\section{Promoting resistance}

Robert Austin and colleagues have developed a microfluidic device that moderates the concentration of antibiotics and nutrients in the environment as a means to simulate a more naturally occurring heterogeneous environment for bacterial isolates, and they follow the emergence of drug resistance in Escherichia coli (Science 333, 1764-1767, 2011). The authors inoculated the microfluidic device, which includes a 96-well array, with wild-type $E$. coli and used two syringe pumps at the top and bottom of the array to establish a concentration gradient of ciprofloxacin. Local microenvironments were created within the wells, in which bacterial density was directly correlated with the ciprofloxacin gradient. Drug resistance emerged quickly, with the rate of development a function of the size of the initial inoculum. The authors demonstrate that the rapid emergence of drug resistance was dependent on the ciprofloxacin gradient, suggesting that the heterogeneous drug environment allowed for a more rapid emergence of drug resistance than did comparable homogenous drug environments. Wholegenome sequencing of the $E$. coli strains from multiple experiments identified four SNPs that were fixed in the drug-resistant bacterial populations. The authors suggest likely functional roles for these mutations in the development of drug resistance.

\section{Repeat expansion in ALS and FTD}

Previous linkage and association studies have implicated a region at chromosome 9p21 as harboring a shared susceptibility locus for amyotrophic lateral sclerosis (ALS) and frontotemporal dementia (FTD). Now, parallel studies by Bryan Traynor and colleagues (Neuron, published online 21 September 2011; doi:10.1016/j.neuron.2011.09.010) and Rosa Rademakers, Ian Mackenzie and colleagues (Neuron, published online 21 September 2011; doi:10.1016/j.neuron.2011.09.011) have identified a hexanucleotide-repeat expansion in a previously uncharacterized gene in the 9p21 region as a major contributor to the familial and sporadic forms of these diseases. Both groups discovered the expansion through sequencing and showed that it segregated with disease in large families with confirmed linkage to the $9 \mathrm{p} 21$ region. They subsequently extended their analyses to familial and sporadic forms of ALS and FTD and found that the expansion was strongly associated with both diseases in multiple case-control collections. In the study by Traynor and colleagues, the expansion was found in $46 \%$ of familial ALS and $21 \%$ of sporadic ALS cases in the Finnish population compared to less than $1 \%$ of controls. The repeat is located in C9orf72, a gene of unknown function expressed broadly in spinal cord and brain tissues, and may contribute to disease risk by promoting the formation of nuclear RNA foci.

$K V$

\section{Epiallele accumulation}

Although a few meiotically heritable epigenetic alleles have been identified in plants, not much is known about how they arise in populations. Joseph Ecker and colleagues now report the identification of spontaneously occurring DNA methylation epialleles in Arabidopsis thaliana mutation accumulation lines (Science, published online 15 September 2011; doi:10.1126/science.1212959). The authors generated base-resolution whole-genome methylation profiles of three ancestral and five descendant mutation accumulation lines. The genomes of all eight lines have been sequenced, allowing the authors to control for genetic variation. The majority of CGs were methylated in all eight lines. Analysis of sites of

Written by Pamela Feliciano, Emily Niemitz, Orli Bahcall \& Kyle Vogan variable methylation showed that ancestral lines are highly similar when considered in terms of both CG single-methylation polymorphisms and differentially methylated regions. The authors identified 72 epialleles that contain methylation variation in the context of CG, CHG and $\mathrm{CHH}$ sequences and determined that these epialleles are not associated with genetic variation. They looked at eight epialleles that overlapped with protein-coding regions and found an association between mRNA expression levels and gain or loss of methylation at four of these loci. This work suggests that transgenerational epigenetic variation may account for more phenotypic diversity than was previously appreciated.

EN

\section{Cntnap2-/- autism model}

The contactin associated protein-like 2 (CNTNAP2) gene has been implicated in autism spectrum disorder (ASD) and cortical dysplasiafocal epilepsy (CFDE) syndrome, which is characterized by seizures, language regression, intellectual disability, hyperactivity and autism. Now Daniel Geschwind and colleagues report the characterization of the mouse knockout of Cntnap2 (Cell 147, 235-246, 2011). Spontaneous seizures were seen in Cntnap $2^{-/-}$mice older than 6 months of age. Cntnap2-deficient animals showed evidence of ectopic neurons in the corpus collosum and neuronal migration defects, similar to the pattern in patients with CDFE syndrome. The authors observed the knockout mice for behavioral abnormalities relevant to ASD and saw evidence of stereotypic motor movements and behavioral inflexibility. Knockout pups emitted fewer isolation-induced ultrasonic vocalizations, which are thought to be distress calls from pups to their mothers, and spent less time interacting with other mice in a juvenile play test. Risperidone is an FDA-approved drug for the alleviation of hyperactivity, repetitive behavior, aggression and selfinjurious behaviors in ASD. Treatment of Cntnap2-deficient mice with risperidone led to lowered activity levels, repetitive grooming behaviors and perseveration, although no effect on social behavior was found. The authors suggest that the Cntnap $2^{-/-}$mouse is an important new tool for assessing potential therapeutics in ASD.

\section{Early metastatic phenotypes}

Cancer has typically been thought of as a linear disease, with metastasis occurring as a late event. However, a few studies suggest that cancer cell dissemination can occur very early in cancer progression. Now, Jean-Pierre Abastado and colleagues use a mouse melanoma model to show that immune cells infiltrate primary tumors and stimulate tumor cell dissemination, providing both a potential explanation for early metastatic phenotypes and a link between inflammation and cancer progression (PLoS Biol. 9, e1001162, 2011). The authors used RETAAD mice that carry an activated RET transgene and spontaneously develop uveal melanomas that eventually progress to cutaneous and visceral metastases. Previous studies have observed increased numbers of a specific subset of immune cells in the blood during tumor progression. Abastado and colleagues observed that these cells preferentially accumulated in primary tumors. The chemokines CXCL1, CXCL2 and CXCL5 were highly expressed in primary tumors and could attract PMNMDSCs in vitro. Depleting mice of PMN-MDSCs showed that these cells favor primary tumor growth, and these mice also had fewer cutaneous metastases, demonstrating that these cells promote metastatic outgrowth. Finally, the authors showed that PMN-MDSCs induce epithelial-mesenchymal transition (EMT), a known hallmark of metastatic cells. 